\title{
3 Research Square \\ Comprehensive Analysis Reveals A Six-Gene Signature and Associated Drugs in Alzheimer Disease
}

\section{Zhengye Jiang}

Xiamen University

\section{Guowei Tan}

Xiamen University and Fujian Medical University Affiliated First Hospital

Zhanxiang Wang ( $\nabla$ jzy0203@qq.com )

\section{Research}

Keywords: Differentially Expressed Genes, Biomarkers, Alzheimer disease, Bioinformatical analysis

Posted Date: May 28th, 2020

DOl: https://doi.org/10.21203/rs.3.rs-30210/v1

License: (9) (i) This work is licensed under a Creative Commons Attribution 4.0 International License. Read Full License 


\section{Abstract}

Background: Alzheimer disease (AD) is a progressive neurodegenerative disease caused by many factors. The essential genes and signaling pathways involved in the pathogenesis of AD are still unknown. The purpose of our research is to analyze and screen out potential molecular biomarkers and related signaling pathways.

Methods: We obtained the gene expression profile of GSE18309 from the gene expression Omnibus website. Then, we used the Limma package in Rstudio to screen out differentially expressed genes (DEGs), followed by the corresponding cell signal pathway enrichment using DAVID analysis. Finally, STRING used to obtain the protein-protein interaction (PPI) network and the corresponding hub gene obtained through MCODE of Cytoscape software.

Results: The results showed that a total of 119 upregulated genes and 160 downregulated genes were identified, which met the criteria of |log2 changes $\mid \geq 2$, adjusted $P$ value $<0.01$. Through the PPI network, the hub gene module we obtained shows that genes such as GNG13, EDNRB, CHRM3, CCKAR, FFAR4 and TRIO are closely related to AD. The signaling pathway is about signal transducer activity, G-protein coupled receptor activity and transmembrane signaling receptor activity.

Conclusions: In summary, the above-obtained hub gene and the identified signaling pathway will help explore the pathogenesis of $A D$; and provide new therapeutic targets and prognostic assessment for AD.

\section{Introduction}

Alzheimer's disease (AD) is a progressive neurodegenerative disease that is gradually known to everyone. Its clinical manifestations are mainly cognitive dysfunction, commonly known as forgetfulness, which eventually leads to Alzheimer's disease. It is also a major fatal disease affecting the elderly One. Numerous evidence in the past show that genetic factors, immune factors, environmental factors, depression, high blood pressure, etc. may be significantly related to the occurrence and development of $A D(1-6)$. At the same time, it is estimated that genetic factors account for the risk of $A D 70 \%(7)$. Although substantial progress has been made in basic experiments and clinical research on AD, its etiology is still unknown. It is worth noting that the treatment of $A D$ can only improve symptoms to a certain extent, and will not hinder the progress of the disease(8). At present, due to the increasing incidence of $A D$ in the elderly and poor prognosis, it is urgent to reveal the etiology and molecular characteristics of $A D$ disease, discover molecular biomarkers of $A D$ disease, and provide the basis for early diagnosis, prevention and $A D$ disease New treatment strategies.

In recent years, high-throughput sequence techniques such as microarray or RNA-seq chip used to analyze differential gene expression and variable splicing variation have been increasingly regarded as essential techniques with significant clinical application prospects in tumor medicine as molecular diagnosis, drug target discovery, prognosis prediction, etc. A public database, the integrated gene expression database (GEO), supported by the national center for biotechnology information (NCBI), 
contains profiles of disease gene expression from dozens of basic experiments. The GEO database is widely used to identify key genes and potential mechanisms for the occurrence of disease and development(9). Although the pathogenesis of $A D$ has been studied in recent years, the pathogenesis and molecular mechanisms of $A D$ progression remain controversial. Therefore, we need to use gene expression chips to export the data from these analyses to modern pathway analysis software, which can find meaningful clues to a new understanding, such as new diagnostic markers and therapeutic targets(10).

In this study, we downloaded one MB microarray dataset (GSE18309) from the Gene Expression Omnibus database (GEO, http://www.ncbi.nlm.nih.gov/geo/), and we used three AD samples and three normal control samples for DEGs analysis. Subsequently, the differentially expressed genes (DEGs) were screened using R software (version 3.6.3) installed Limma packages $(11,12)$; later, Venny online tool was used for further comprehensive analysis. Then gene ontology (GO) and pathway enrichment were analyzed on DAVID's website (https://david.\%20ncifcrf.gov)(13). By analyzing their critical cellular signaling pathways and biological functions, a string database was used to generate protein-protein interaction networks (PPI). In summary, the above analysis identified several essential AD related genes and pathways and further identified potential candidates for diagnostic, prognostic, and therapeutic treatment.

\section{Results}

\section{DEGs identification}

In this study, we included three $A D$ samples and three normal control samples for the analysis to identify DEGs linked with AD. The GSE18309 was analyzed using Rstudio software, and the following DEG sets were determined. Using $\mid \log 2$ fold change $(F C) \mid \geq 2$ criteria and adjusted $P$ values $<0.01$, a total of 279 DEGs were identified from the GSE62600 data set, including 119 upward adjustments and 160 downward adjustments (Table 1). 
Table 1

279 Differentially Expressed Genes (DEGs) were identified from GSE62600, including 119 upregulated genes and 160 downregulated genes in the patients with Alzheimer disease, compared to controla ${ }^{\text {. }}$

\begin{tabular}{|c|c|}
\hline DEGs & Gene name \\
\hline $\begin{array}{l}\text { Upregulated } \\
\text { genes }\end{array}$ & $\begin{array}{l}\text { LINC00664, PMP2, FAM170B, CLDN11, VPS33A, RTP3, HIF3A, SLC4A1, RBM43, } \\
\text { TTC28-AS1, KRT17P5, AHNAK, SLC17A8, MAP2K1, FREM1, HSD17B12, ABCC11, } \\
\text { MYH3, KITLG, OR9A1P, CDC42BPA, PPP5D1, TNS1, TPM1, GJA10, EGLN2, OPRM1, } \\
\text { SMAD5-AS1, TRABD2A, FOXO3, MAATS1, GPR161, CASC15, IFNW1, ADGRD2, } \\
\text { DLGAP1, GNG13, ALDH4A1, MEG3, MEP1A, ARPP21, SLC6A8, VWDE, ANKUB1, } \\
\text { CXCL3, DAZAP2, NTNG1, GPX8, C110rf96, ZNF425, TBX5, UST-AS1, ZNF135, } \\
\text { KCNQ1, DAOA-AS1, ASCL 1, METAP1D, C21Orf58, SUCLG2-AS1, NRP2, ANKRD29, } \\
\text { SORBS2, ITIH3, KDSR, IL22RA2, POLN, SPINK13, FZD10, AGAP11, ANKRD13C, } \\
\text { DOCK6, TRIO, CACNA2D1, ALAS2, SYN2, SARM1, RAD51B, RBPMS2, GRIP2, BTG4, } \\
\text { FGF14, CRMP1, KCNE4, LRP12, UNC13C, FITM2, RPL35A, CRHR1, PRO2958, } \\
\text { HMGA2, C1Orf101, KCNMB2, PDIA2, COL 5A1, CYP2A13, PPEF1, RAG2, PDE1C, } \\
\text { CLCN7, CALCRL, CHRM3, BGLT3, SOX6, SLC6A11, ZNF462, GPR158, CDH8, DIRC1, } \\
\text { CXCL 11, TMCO5B, SOBP, DPPA5, TEAD4, SEMA6D, HTN3, MAMDC2, HGD, LMOTDN } \\
\text { PDLIM3 }\end{array}$ \\
\hline
\end{tabular}

Downregulated ZMYND11, ARL10, EGFEM1P, FFAR4, CAPRIN2, KIAA1462, PAQR9, EMP2, CYMP, PGM5, HLA-DOA, TMPRSS5, PREX2, NARF, M4SF20, LAMB4, CAMK2N1, CAPN13, genes MTMR1, PLCE1, SMARCA2, PCDH7, COL4A5, MAGEA4, BNC1, SNORD8, CLIP1-AS1, FBXO4, SERTAD4, CEP152, DNMBP, EDNRB, POLR3G, LMBRD2, ITGB2, GIPC3, TPD52L 1, BTN1A1, ILDR2, MAP1B, PDE4A, CES1P1, IGFBP2, C2CD2L, TMEM217, DSEL, RCBTB2, ADCY10, MIR670HG, CSRP2, ZNF233, KCNK1, IL 17A, PLEKHB1, CACNA1B, PDLIM4, TAT, ACTG1P17, CBR3-AS1, GPRC5B, LAYN, ITGA11, NAALAD2, ATXN7L1, UNC80, SLC3OA7, SRRM5, GJC1, ZNF81, POU3F2, SPATA45, TLE1, M1AP, RHOJ, DAW1, LRRC34, NKAPP1, ARR3, TBC1D16, CRNN, INTS1, CRISP1, P2RX6, ZIC5, SYNPO2, SPON1, COX11, PER4, SERPINB13, TONSL, 730101, SPANXA2-OT1, SULT1E1, PMCHL2, EPS8L2, ZNF519, RASEF, DNAH6, GSTA3, TNXB, COL 10A1, JUN, LARGE-AS1, FLRT3, MICALL2, CDKN2D, CETN1, MUC3B, C7orf77, NOX1, MAGI1, KIAA0087, FOXI1, ANKRD53, NLGN4X, CABP2, FOXP3, TLDC1, ACVR1B, CCKAR, ATP8B3, WNT5A, LAMA4, CEP70, PPARGC1B, PRSS35, CENPA, C9orf116, KIAA1549, LIMK1, AMFR, CCNY, CASD1, ZNF214, ADAM22, LACE1, MYO7B, ZNF709, IL6ST, STX19, TPT1-AS1, FCGR2C, PATJ, DENND5B, ZNF264, PREPL, GABRA2, DNAJB8-AS1, NDST4, AKR1D1, SAV1, PER2, PCSK2, PHKG1, ZNF595, LRRTM1, TRPC2, FAM64A, CSPG4, CNBD1

aThe upregulated genes were listed from the largest to the smallest of fold changes, and downregulated genes were listed from the smallest to largest.

\section{Function And Signal Pathway Enrichment Analysis}

To further explore the potential significance of these identified DEGs in MB, We upload the filtered DEGs to the DAVID website and set the key standard to $P<0: 05$ to analyze and identify $G 0$ terms and KEGG pathway, which was divided into three functional groups: cell component (CC), molecular function (MF), and biological process (BP). The results in Fig. 2 show the following six most important terms: BP, CC, and MF for DEGs, respectively. We also show the annotations of upregulated genes and downregulated genes in detail. As shown in Table 2, within the BP, the upregulated DEGs were mainly related to the regulation of voltage-gated calcium channel activity, regulation of ion transmembrane transport, and cellcell signaling, and the downregulated DEGs were primarily related to the regulation of phosphorylation, 
regulation of protein modification process, and regulation of protein phosphorylation. Within the $\mathrm{CC}$, the upregulated DEGs were mainly related to contractile fiber part, myofibril, and contractile fiber, and the downregulated DEGs were mainly related to cell junction, dendrite, and neuron part. Within the MF, the upregulated DEGs were mainly related to heparin binding, structural constituent of muscle, and voltagegated ion channel activity, and the downregulated DEGs were mainly related to protein kinase regulator activity, protein complex binding, and kinase regulator activity. The cellular signaling pathway represented by 279 DEGs was analyzed by KEGG. Figure 3 shows the most significant enrichment pathways for DEGs. And Table 2 lists the significant enrichment pathways for the downregulation of DEGs, while there are no available significantly enriched pathways of the upregulated DEGs. Among them, the downregulated DEGs were mainly enriched in the ECM-receptor interaction, Amoebiasis, and Calcium signaling pathway. 
Table 2

The significant gene ontology and KEGG enrichment terms of Upregulated and downregulated DEGs, respectively

\begin{tabular}{|c|c|c|c|}
\hline Category & Term & Count & $P$ Value \\
\hline \multicolumn{4}{|l|}{ Upregulated DEGs } \\
\hline GOTERM_BP_FAT & $\begin{array}{l}\text { GO: } 1901385 \sim \text { regulation of voltage-gated calcium } \\
\text { channel activity }\end{array}$ & 4 & $5.55 \mathrm{E}-04$ \\
\hline GOTERM_BP_FAT & $\begin{array}{l}\text { GO:0034765 regulation of ion transmembrane } \\
\text { transport }\end{array}$ & 9 & 0.001566964 \\
\hline GOTERM_BP_FAT & GO:0007267 cell-cell signaling & 19 & 0.001651788 \\
\hline GOTERM_CC_FAT & Go:0044449 contractile fiber part & 6 & 0.00737112 \\
\hline GOTERM_CC_FAT & GO:0030016 myofibril & 6 & 0.008457733 \\
\hline GOTERM_CC_FAT & GO:0043292 contractile fiber & 6 & 0.010571241 \\
\hline GOTERM_MF_FAT & G0:0008201 heparin binding & 5 & 0.014668566 \\
\hline GOTERM_MF_FAT & G0:0008307 structural constituent of muscle & 3 & 0.025277132 \\
\hline GOTERM_MF_FAT & Go:0005244 voltage-gated ion channel activity & 5 & 0.025293473 \\
\hline \multicolumn{4}{|l|}{$\begin{array}{l}\text { Downregulated } \\
\text { DEGs }\end{array}$} \\
\hline GOTERM_BP_FAT & G0:0042325 regulation of phosphorylation & 23 & 3.63E-04 \\
\hline GOTERM_BP_FAT & $\begin{array}{l}\text { G0:0031399 regulation of protein modification } \\
\text { process }\end{array}$ & 25 & 7.10E-04 \\
\hline GOTERM_BP_FAT & GO:0001932 regulation of protein phosphorylation & 21 & $9.64 \mathrm{E}-04$ \\
\hline GOTERM_CC_FAT & GO:0030054 cell junction & 23 & 3.39E-04 \\
\hline GOTERM_CC_FAT & GO:0030425 dendrite & 10 & 0.007006 \\
\hline GOTERM_CC_FAT & G0:0097458 neuron part & 19 & 0.007583 \\
\hline GOTERM_MF_FAT & GO:0019887 protein kinase regulator activity & 6 & 0.009196 \\
\hline GOTERM_MF_FAT & GO:0032403 protein complex binding & 13 & 0.011479 \\
\hline GOTERM_MF_FAT & Go:0019207 kinase regulator activity & 6 & 0.013991 \\
\hline KEGG_PATHWAY & hsa04512:ECM-receptor interaction & 5 & 0.002395 \\
\hline KEGG_PATHWAY & hsa05146:Amoebiasis & 5 & 0.004886 \\
\hline KEGG_PATHWAY & hsa04020:Calcium signaling pathway & 6 & 0.005933 \\
\hline
\end{tabular}




\section{Module Screening From The Ppi Network}

DEGs were analyzed by STRING online database (http://string-db.org) and Cytoscape software, a total of 279 DEGs (119 upregulated and 160 downregulated genes) were screened into the DEG PPI network complex, including 42 nodes with 44 edges and a score of $>0.900$ (highest confidence) (Fig. 4A). Afterward, based on MCODE, select the prominent modules in the PPI network (6 nodes, 15 edges, Fig. 4B), which three upregulated genes (GNG13, CHRM3, and TRIO), and three downregulated genes (GNG13, CCKAR, and FFAR4).

\section{Drug-gene Interaction And Functional Analysis Of Potential Genes}

To get interaction between genes and the existing drugs and explore the potential application of the new drug indications for a human hernia. The drug-gene interaction database (DGIdb: https://www.dgidb.org) is an open-source and supports searching, browsing, and filtering of information on drug-gene interactions based on over thirty trusted sources(47). As the potential targets, the module genes were pasted into the drug-gene database to search for existing drugs or compounds. These potential genes which have matched drugs were obtained and also performed functional enrichment analysis.

\section{Discussion}

It is well known that $A D$ is a genetically complex neurodegenerative disease characterized by the formation of extracellular senile plaques amyloid- $\beta(A \beta)$ peptides, intracellular neurofibrillary tangles (non-functional testing), and structure Brain regions related to functional changes and memory(14-16), Alzheimer's disease affects the quality of life of patients, is not conducive to the lives of patients, and places a heavy burden on patients' families and the entire society. However, at present, the pathogenesis of $A D$ and effective treatments for $A D$ patients is rare. Therefore, in this study, we hope to screen out key candidate genes and signaling pathways of early AD. We found 119 upregulated DEGs and 160 downregulated DEGs by comparing the three AD sample with three normal control samples. Through $G O$, KEGG, and PPI network analysis, we have identified hub genes such as GNG13, EDNRB, CHRM3, CCKAR, TRIO, and FFAR4, coupled with the ECM-receptor interaction signaling pathway have been identified.

We have listed these selected genes. In previous studies, it was only found that the disorder of GNG13 is related to breast cancer disease plan(17). In the study of sun(18) and others, it was found that GNG13 may play an essential role in the development of prostate cancer. EDNRB's involvement in the event of nerve cells and glial cells has been confirmed in fetal and adult brains. EDNRB activation in the brain increases the proliferation of neurons and astrocytes, increases the expression of cytoskeletal proteins in astrocytes, and increases neurotrophic factors' production by astrocytes(19). These results indicate that the loss of EDNRB function leads to a significant decrease in cell proliferation and increased apoptosis in all subregions of the hippocampus and dentate gyrus. EDNRB plays an vital role in the proliferation of 
normal cells in the rat hippocampus(20). The study of Schmidt et al. found that CCKAR has a certain correlation with schizophrenia and is related to the regulation of ischemia-hypoxia(21). Evidence suggests that FFAR4 has a role in regulating energy balance, including controlling blood sugar and intestinal hormone secretion(22). Current research shows that sustained FFAR4 stimulation in the brain can reduce anxiety behaviors, thus indicating that FFAR4 acts as a potential pathway through omega-3 $(\omega-3)$ polyunsaturated fatty acids (PUFA) central anti-anxiety behavior(23). It is recognized the that TRIO plays an important role in cell division, cell migration, and other functions and plays a role in synapse formation by regulating excitatory synaptic transmission(24-26). In previous mouse experiments, it was found that the lack of heterozygosity or homozygosity of TRIO in the hippocampus will lead to progressive defects in their learning and social skills(27-29).

As for the CHRM3 gene, which is a cholinergic receptor and is related to schizophrenia, it may be a potential therapeutic target for COPD patients $(30,31)$. And we found six antipsychotic drugs targeting CHRM3. Aripiprazole is an atypical antipsychotic drug approved for schizophrenia in adults and adolescents, mania in children and adults with bipolar disorder, autism, and major depression in adults. In several experiments, it was found that aripiprazole is more effective in controlling behavioral symptoms in $A D$ patients $(32,33)$. The results of the cohort study experiments by Vigen et al. Showed that $A D$ patients treated with olanzapine had a significant decrease in the cognitive summary score and mini mental state examination (MMSE). The effect is obvious(34). At the same time, in the study of Zheng et al., It was found that aripiprazole combined with olanzapine was effective in treating elderly Alzheimer's disease with mental disorders. It promotes the recovery of nerve function and produces a lower incidence of adverse reactions(35). In another experiment, it was found that the treatment of AD mice with CLOZAPINE can improve memory impairment and $A \beta$ generation during the formation of amyloid(36). Promethazine is one of the most commonly used antipsychotic drugs(37), but there is no relevant report showing the correlation between promethazine and AD. At the same time, we searched the literature to find that CHLORPROTHIXENE and LEVOMEPROMAZINE have any connection with the treatment of AD, and the previous research of the first four drugs seems to remind us that the two may play some role in the development of $A D$ Unknown role.

In short, in this article, we explored potential essential candidate genes and critical signaling pathways for DEGs in the occurrence and development of AD. Through commonly used analysis methods, key candidate genes and signal pathways are gradually screened by the analysis sequence of DEG, GO, KEGG, and PPI. Then through drug-gene interaction analysis, we identified six existing antipsychotic drugs. Our research has improved our understanding of the occurrence mechanism and potential molecular mechanisms of $A D$; these selected candidate genes, signaling pathways, and potential therapeutic drugs may provide us with clues for new AD diagnostic strategies, targeted therapy, and prognostic analysis. However, to determine whether these genes are related to the occurrence and development of $A D$ and whether these drugs can delay or prevent the progress of $A D$, it needs to be further confirmed by molecular biology, cell experiments, and even clinical trials. This study has certain limitations. For example, the lack of correlation analysis between these genes and clinical information requires basic experiments to explore the role of these genes in $A D$. 


\section{Conclusions}

Through applying a series of bioinformatics methods to gene expression profiling, we acquired 6 potential biomarkers (GNG13, CHRM3, TRIO, GNG13, CCKAR, and FFAR4) and six antipsychotics drug (ARIPIPRAZOLE, OLANZAPINE, CLOZAPINE, PROMAZINE, CHLORPROTHIXENE and LEVOMEPROMAZINE), which will provide insight for new study targets and new drug indications.

\section{Methods}

\section{Data Collection}

From the National Biotechnology Information Center (NCBI) Gene Expression Comprehensive (GEO) Database $(9,38)$, we downloaded the gene expression chip data GSE18309(39) we need to analyze. The data GSE18309 was uploaded by Kuang-Den Chen et al., using the Affymetrix GPL570 platform (Affymetrix Human Genome U133 Plus 2.0 Array) as a reference. We selected three AD samples and three normal control samples to analyze and identify the hub gene and signaling pathway.

\section{Data Preprocessing}

After obtaining GSE18309, firstly, the obtained probe identification numbers (IDs) are converted into gene symbols or translators by $\mathrm{R}$ software. Then, the same gene should be processed corresponding to multiple probes, and the most significant expression value is taken as the gene expression value. Next, the non-mRNA probe is deleted from it. Finally, through the Affy package, the obtained gene expression value was normalized, and the signal intensity of the gene was converted by log2 and normalized $(38,40)$.

\section{Identification Of Degs}

We use linear models to evaluate differential expressions and to analyze design experiments. Use the linear models for microarray data (limma) package in R software (version 3.6.3) to identify the DEGs based on a series of matrix files and respectively divide into upregulated genes and downregulated genes. Significant DEGs were selected for further analysis by cut-off criterion (|log2 variation $(F C) \mid \geq 2$ and adjusted $P$ value $<0.01)(41)$.

\section{Gene Ontology Analysis Of Degs And Kegg Pathway Analysis}

Gene Ontology (GO) provides a standard vocabulary of corresponding terms with annotations that illuminate the characteristics of genetic products. The $\mathrm{GO}$ term reflects the current understanding of genes in terms of biological processes (BP), cell composition (CC), and molecular function (MF) $(42,43)$. 
Also, the Kyoto Encyclopedia of Genes and Genomes (KEGG)(44) provides a large number of data resources of known biological pathways, which are annotated as a gene or a group of genes/proteins with their respective KEGG pathways. To interpret the function and signal pathway analysis of DEGs, we use a variety of online tools for functional and pathway enrichment analysis(13). For example, DAVID is an online site that provides genetic annotations, visualizations, and genetic attributes. $P<0.05$ was considered to be statistically significant.

\section{Module analysis, identification of the hub gene, and analysis of the PPI network of the DEGs}

The search tool (STRING) was used to demonstrate DEG-encoded protein and protein-protein interaction (PPI) information(45), database for retrieving interacting genes. At first, to assess the interaction between DEGs, we mapped the list of DEGs to the STRING website. Second, the PPIs of DEGs with a comprehensive score of $>0.9$ (medium confidence) and genes closely related to other genes, and the degree of selection $\geq 10(46)$. After that, PPI networks were visualized using Cytoscape, and hub genes were identified according to the degree of connectivity between DEGs. The MCODE parameters criteria were set by default, except K-core $=5$. Besides, the functional enrichment analysis of DEGs of each module was carried out with $\mathrm{P}<0.05$ as the cut-off standard.

\section{Statistics Analysis}

The moderate t-test was applied to identify DEGs; Fisher Exact test was used to analyzed GO and KEGG annotation enrichments. All statistical analyses were executed in R version 3.6.3 software Fig. 1

\section{Declarations}

\section{Funding}

No funding was received for this article.

\section{Consent for publication}

All authors agreed to publish

\section{Availability of data and material}

All data are repeatable

\section{Authors' contributions}

Conception and design of the research: Zhengye Jiang; acquisition of data: Zhengye Jiang; analysis and interpretation of data: Guowei Tan; statistical analysis: Tanguo Wei; drafting the manuscript: Zhengye Jiang; revision of manuscript for important intellectual content: Zhanxiang Wang. All authors read and approved the final manuscript. 
Acknowledgements

None

\section{Availability of data and material}

The datasets used and analysed during the current study are available from the corresponding author on reasonable request.

\section{Competing interests}

The authors declare that they have no competing interests

\section{Ethics approval and consent to participate}

None

\section{References}

1. Cacabelos R, Meyyazhagan A, Carril JC, Cacabelos P, Teijido O. Pharmacogenetics of Vascular Risk Factors in Alzheimer's Disease. Journal of Personalized Medicine. 2018;8(1).

2. Jayaraman A, Pike CJ. Alzheimer's Disease and Type 2 Diabetes: Multiple Mechanisms Contribute to Interactions. Current Diabetes Reports. 2014;14(4).

3. Reitz C. Genetic diagnosis and prognosis of Alzheimer's disease: challenges and opportunities. Expert Rev Mol Diagn. 2015;15(3):339-48.

4. Rivera DS, Inestrosa NC, Bozinovic F. On cognitive ecology and the environmental factors that promote Alzheimer disease: lessons from Octodon degus (Rodentia: Octodontidae). Biological Research. 2016;49.

5. Tolppanen AM, Taipale H, Hartikainen S. Head or brain injuries and Alzheimer's disease: A nested case-control register study. Alzheimer's dementia: the journal of the Alzheimer's Association. 2017;13(12):1371-9.

6. Vijayan M, Reddy PH. Stroke. Vascular Dementia, and Alzheimer's Disease: Molecular Links. Journal of Alzheimers Disease. 2016;54(2):427-43.

7. Girard H, Potvin O, Nugent S, Dallaire-Theroux C, Cunnane S, Duchesne S, et al. Faster progression from $\mathrm{MCl}$ to probable $\mathrm{AD}$ for carriers of a single-nucleotide polymorphism associated with type 2 diabetes. Neurobiology of Aging. 2018;64.

8. Graham WV, Bonito-Oliva A, Sakmar TP. Update on Alzheimer's Disease Therapy and Prevention Strategies. In: Caskey CT, editor. Annual Review of Medicine, Vol 68. Annual Review of Medicine. 682017. p. $413-30$.

9. Barrett T, Wilhite SE, Ledoux P, Evangelista C, Kim IF, Tomashevsky M, et al. NCBI GEO: archive for functional genomics data sets-update. Nucleic Acids Res. 2013;41(D1):D991-D5. 
10. Guo Y, Bao Y, Ma M, Yang W. Identification of Key Candidate Genes and Pathways in Colorectal Cancer by Integrated Bioinformatical Analysis. International Journal of Molecular Sciences. $2017 ; 18(4)$.

11. Smyth GK. Limma: Linear models for microarray data. In: Gentalman R, Carey VJ, Huber W, Irizarry RA, Dudoit S, editors Bioinformatics and Computational Biology Solution Using R and Bioconductor. Statistics for Biology and Health2005. p. 397-420.

12. Racine JS. RStudio:. A Platform-Independent IDE for R and Sweave. J Appl Econom. 2012;27(1):167-72.

13. Huang DW, Sherman BT, Lempicki RA. Systematic and integrative analysis of large gene lists using DAVID bioinformatics resources. Nat Protoc. 2009;4(1):44-57.

14. Eimer WA, Kumar DVK, Shanmugam NKN, Rodriguez AS, Mitchell T, Washicosky KJ, et al. Alzheimer's Disease-Associated beta-Amyloid Is Rapidly Seeded by Herpesviridae to Protect against Brain Infection. Neuron. 2018;99(1):56-+.

15. Forner S, Baglietto-Vargas D, Martini AC, Trujillo-Estrada L, LaFerla FM. Synaptic Impairment in Alzheimer's Disease: A Dysregulated Symphony. Trends Neurosci. 2017;40(6):347-57.

16. Bakota L, Brandt R. Tau Biology and Tau-Directed Therapies for Alzheimer's Disease. Drugs. 2016;76(3):301-13.

17. Fu Y, Kadioglu O, Wiench B, Wei Z, Wang W, Luo M, et al. Activity of the antiestrogenic cajanin stilbene acid towards breast cancer. J Nutr Biochem. 2015;26(11):1273-82.

18. Sun J, Li S, Wang F, Fan C, Wang J. Identification of key pathways and genes in PTEN mutation prostate cancer by bioinformatics analysis. Bmc Medical Genetics. 2019;20(1).

19. Shinohara H, Udagawa J, Morishita R, Ueda H, Otani H, Semba R, et al. G(i2) signaling enhances proliferation of neural progenitor cells in the developing brain. J Biol Chem. 2004;279(39):41141-8.

20. Xie D, Croaker GDH, Li J, Song Z-M. Reduced cell proliferation and increased apoptosis in the hippocampal formation in a rat model of Hirschsprung's disease. Brain Res. 2016;1642:79-86.

21. Schmidt-Kastner R, van Os J, Steinbusch HWM, Schmitz C. Gene regulation by hypoxia and the neurodevelopmental origin of schizophrenia. Schizophr Res. 2006;84(2-3):253-71.

22. Ulven T, Christiansen E. Dietary Fatty Acids and Their Potential for Controlling Metabolic Diseases Through Activation of FFA4/GPR120. In: Bowman BA, Stover PJ, editors. Annual Review of Nutrition, Vol 35. Annual Review of Nutrition. 352015. p. $239-63$.

23. Auguste S, Fisette A, Fernandes MF, Hryhorczuk C, Poitout V, Alquier T, et al. Central Agonism of GPR120 Acutely Inhibits Food Intake and Food Reward and Chronically Suppresses Anxiety-Like Behavior in Mice. International Journal of Neuropsychopharmacology. 2016;19(7).

24. Iyer SC, Wang D, lyer EP, Trunnell SA, Meduri R, Shinwari R, et al. The RhoGEF trio functions in sculpting class specific dendrite morphogenesis in Drosophila sensory neurons. PLoS One. 2012;7(3):e33634. 
25. Cannet A, Schmidt S, Delaval B, Debant A. Identification of a mitotic Rac-GEF, Trio, that counteracts MgcRacGAP function during cytokinesis. Molecular biology of the cell. 2014;25(25):4063-71.

26. Schmidt S, Debant A. Function and regulation of the Rho guanine nucleotide exchange factor Trio. Small GTPases. 2014;5:e29769.

27. Zong W, Liu S, Wang X, Zhang J, Zhang T, Liu Z, et al. Trio gene is required for mouse learning ability. Brain Res. 2015;1608:82-90.

28. Tao T, Sun J, Peng Y, Wang P, Chen X, Zhao W, et al. Distinct functions of Trio GEF domains in axon outgrowth of cerebellar granule neurons. Journal of genetics genomics $=$ Yi chuan xue bao . 2019;46(2):87-96.

29. Katrancha SM, Shaw JE, Zhao AY, Myers SA, Cocco AR, Jeng AT, et al. Trio Haploinsufficiency Causes Neurodevelopmental Disease-Associated Deficits. Cell reports. 2019;26(10):2805-17.e9.

30. Wain LV, Shrine N, Artigas MS, Erzurumluoglu AM, Noyvert B, Bossini-Castillo L, et al. Genome-wide association analyses for lung function and chronic obstructive pulmonary disease identify new loci and potential druggable targets. Nat Genet. 2017;49(3):416-25.

31. Wang Q, Cheng W, Li M, Ren H, Hu X, Deng W, et al. The CHRM3 gene is implicated in abnormal thalamo-orbital frontal cortex functional connectivity in first-episode treatment-naive patients with schizophrenia. Psychol Med. 2016;46(7):1523-34.

32. Streim JE, Porsteinsson AP, Breder CD, Swanink R, Marcus R, McQuade R, et al. A randomized, double-blind, placebo-controlled study of aripiprazole for the treatment of psychosis in nursing home patients with Alzheimer disease. American Journal of Geriatric Psychiatry. 2008;16(7):537-50.

33. Mintzer JE, Tune LE, Breder CD, Swanink R, Marcus RN, McQuade RD, et al. Aripiprazole for the treatment of psychoses in institutionalized patients with Alzheimer dementia: A multicenter, randomized, double-blind, placebo-controlled assessment of three fixed doses. American Journal of Geriatric Psychiatry. 2007;15(11):918-31.

34. Vigen CLP, Mack WJ, Keefe RSE, Sano M, Sultzer DL, Stroup TS, et al. Cognitive Effects of Atypical Antipsychotic Medications in Patients With Alzheimer's Disease: Outcomes From CATIE-AD. Am J Psychiatry. 2011;168(8):831-9.

35. Zheng N, Wang N, Jia J-M. Therapeutic benefit of aripiprazole-olanzapine combination in the treatment of senile Alzheimer's disease complicated by mental disorders. Tropical Journal of Pharmaceutical Research. 2020;19(2):441-6.

36. Choi Y, Jeong HJ, Liu QF, Oh ST, Koo B-S, Kim Y, et al. Clozapine Improves Memory Impairment and Reduces A beta Level in the Tg-APPswe/PS1dE9 Mouse Model of Alzheimer's Disease. Mol Neurobiol. 2017;54(1):450-60.

37. Nobili A, Pasina L, Trevisan S, Riva E, Lucca U, Tettamanti M, et al. Use and misuse of antipsychotic drugs in patients with dementia in Alzheimer special care units. Int Clin Psychopharmacol. 2009;24(2):97-104.

38. Edgar R, Domrachev M, Lash AE. Gene Expression Omnibus: NCBI gene expression and hybridization array data repository. Nucleic Acids Res. 2002;30(1):207-10. 
39. Hooper CM, Hawes SM, Kees UR, Gottardo NG, Dallas PB. Gene Expression Analyses of the SpatioTemporal Relationships of Human Medulloblastoma Subgroups during Early Human Neurogenesis. Plos One. 2014;9(11).

40. Larriba Y, Rueda C, Fernandez MA, Peddada SD. Microarray Data Normalization and Robust Detection of Rhythmic Features. In: BolonCanedo V, AlonsoBetanzos A, editors Microarray Bioinformatics. Methods in Molecular Biology. 19862019. p. 207-25.

41. Reiner A, Yekutieli D, Benjamini Y. Identifying differentially expressed genes using false discovery rate controlling procedures. Bioinformatics. 2003;19(3):368-75.

42. Hale ML, Thapa I, Ghersi D. FunSet: an open-source software and web server for performing and displaying Gene Ontology enrichment analysis. Bmc Bioinformatics. 2019;20.

43. Carbon S, Dietze H, Lewis SE, Mungall CJ, Munoz-Torres MC, Basu S, et al. Expansion of the Gene Ontology knowledgebase and resources. Nucleic Acids Res. 2017;45(D1):D331-D8.

44. Kanehisa M, Goto S. KEGG: Kyoto Encyclopedia of Genes and Genomes. Nucleic Acids Res. 2000;28(1):27-30.

45. Franceschini A, Szklarczyk D, Frankild S, Kuhn M, Simonovic M, Roth A, et al. STRING v9.1: proteinprotein interaction networks, with increased coverage and integration. Nucleic Acids Res. 2013;41(D1):D808-D15.

46. Zhou Z, Cheng Y, Jiang Y, Liu S, Zhang M, Liu J, et al. Ten hub genes associated with progression and prognosis of pancreatic carcinoma identified by co-expression analysis. International Journal of Biological Sciences. 2018;14(2):124-36.

47. Griffith M, Griffith OL, Coffman AC, Weible JV, McMichael JF, Spies NC, et al. DGIdb: mining the druggable genome. Nat Methods. 2013;10(12):1209-+.

\section{Figures}



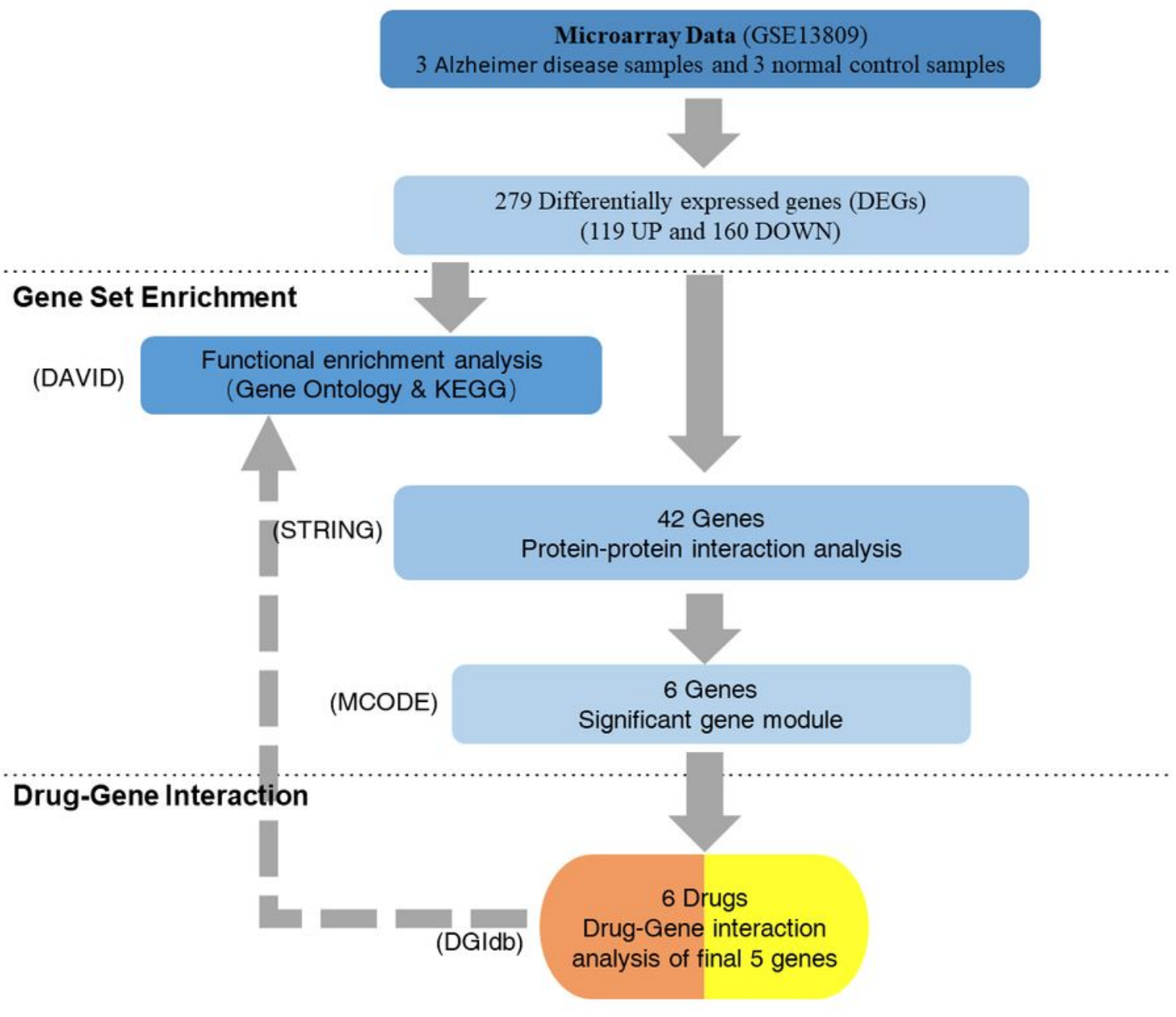

\section{Figure 1}

The framework of data analyses 


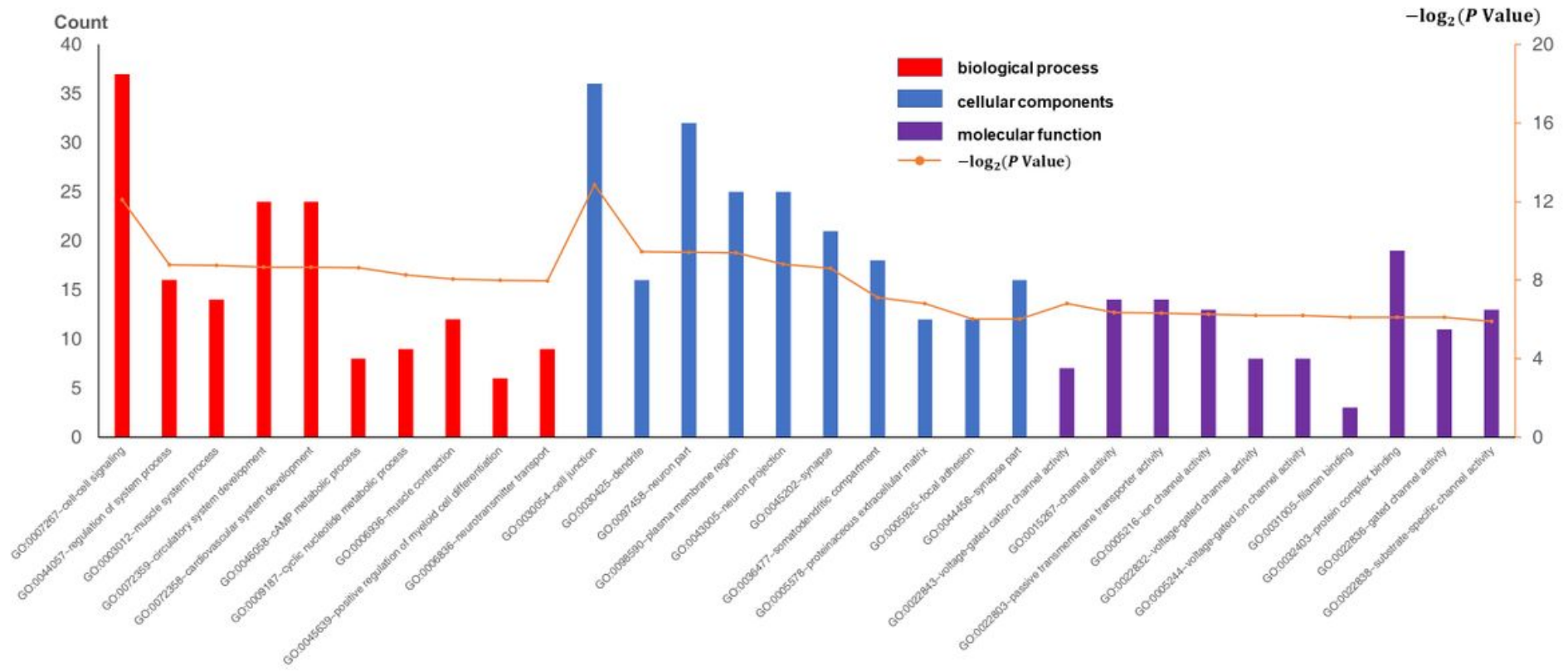

Figure 2

All available significant gene ontology enrichment terms of the differentially expressed genes (DEGs).

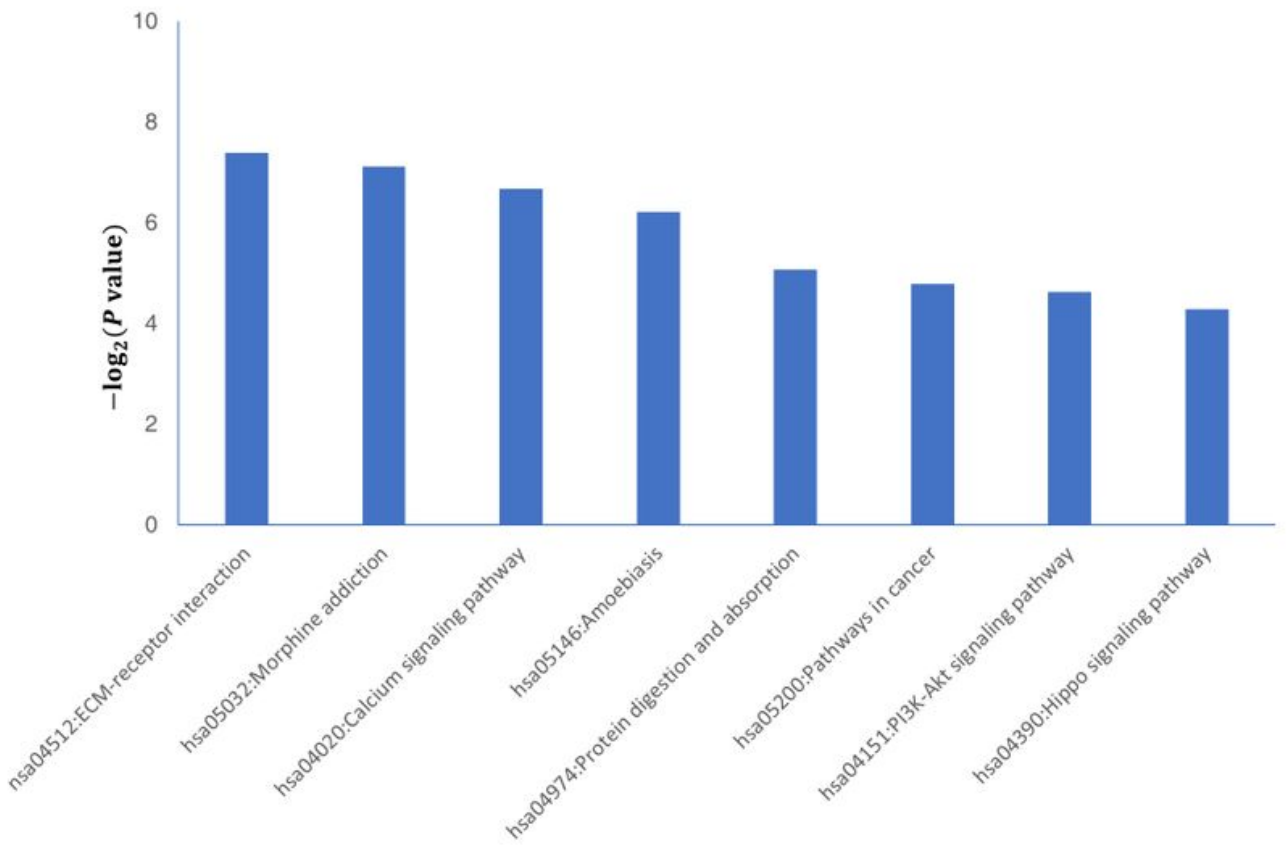


Figure 3

Significantly enriched signal pathway of differentially expressed genes (DEGs) in Alzheimer disease.
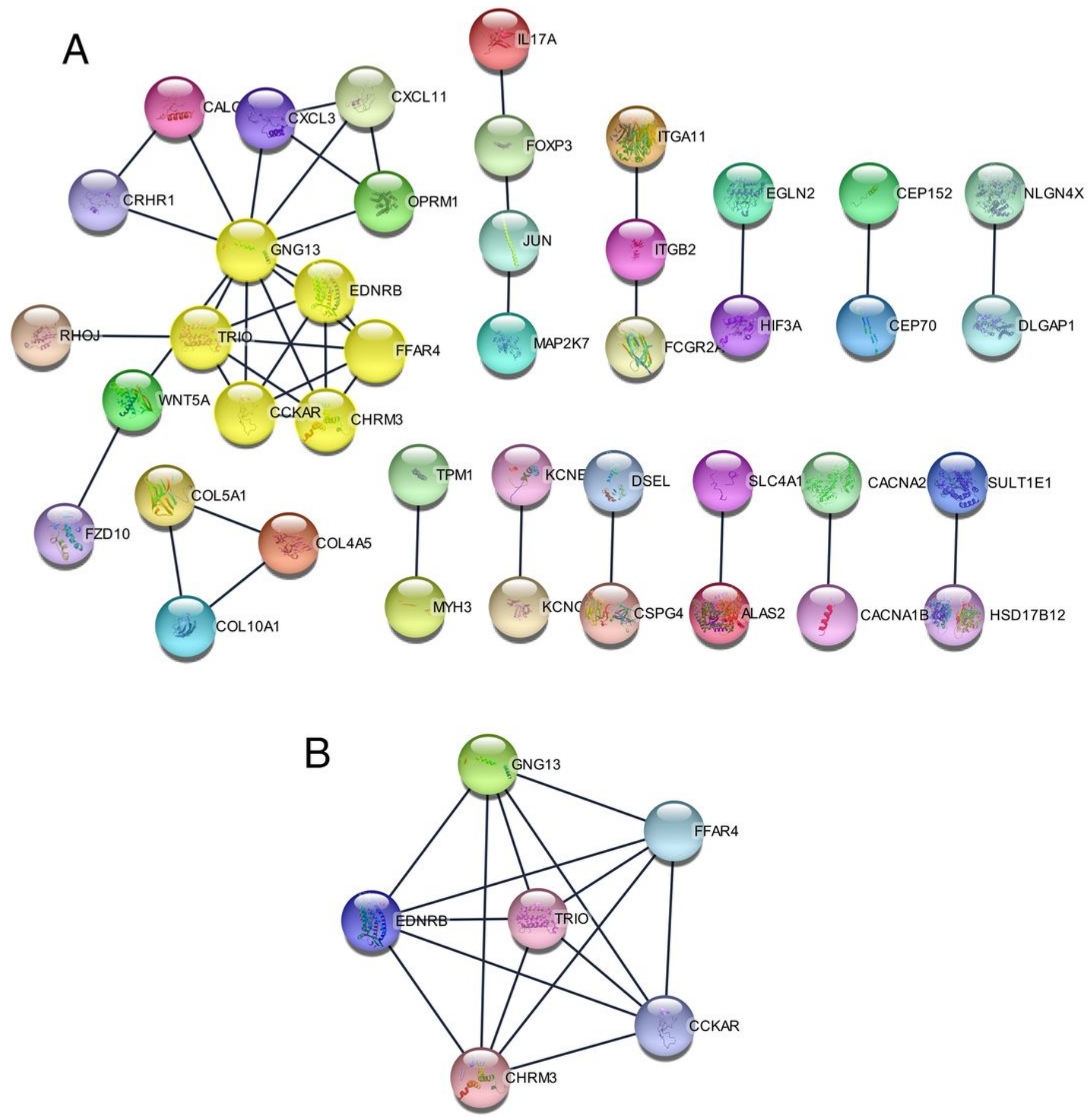

Figure 4

The protein-protein interaction (PPI) networks construction and significant gene modules analysis. 